\title{
Diferentes Metodologias de Dosagem de Misturas Solo-RAP para Uso em Pavimentação
}

\author{
Different Methodologies for Dosing soil-RAP Mixes for Use in Paving
}

\author{
Victor Garcia de Almeida ${ }^{1}$ (), Mario Sergio de Souza Almeida ${ }^{1}$ (1), \\ Fernanda Costa da Silva Maciel ${ }^{1}$ (1), Luciana Negrão de Moura ${ }^{1}$ (1), \\ Weiner Gustavo Silva Costa ${ }^{1}$ (1) \& Maria do Socorro Costa São Mateus ${ }^{2}$
}

\author{
'Universidade Federal do Recôncavo da Bahia, Centro de Ciências Exatas e Tecnológicas, Av. Rui Barbosa, 710, 44380-000, \\ Cruz das Almas, Bahia, Brasil \\ 2Universidade Estadual de Feira de Santana, Departamento de Tecnologia, Programa de Pós-Graduação em Engenharia Civil \\ e Ambiental, Av. Transnordestina, s/n, 44036900, Feira de Santana, Bahia, Brasil \\ E-mails: victor_ma12@hotmail.com; mario.almeida@ufrb.edu.br; nandamaciel_@hotmail.com; luciananegrao@hotmail.com; \\ weiner@ufrb.edu.br; somateus@gmail.com
}

\section{Resumo}

O crescente aproveitamento de resíduos na Engenharia Rodoviária vem ganhando força em vários países, incluindo o Brasil, em função dos ganhos ambientais e econômicos associados a esta atividade. A presente pesquisa buscou avaliar misturas de solo e RAP (Reclaimed Asphalt Pavement), para utilização como camada de pavimentos rodoviários, a partir de ensaios de Índice de Suporte Califórnia (ISC) e ensaios de Resistência à Compressão Não Confinada (RCNC). O estudo avaliou a influência de diferentes teores de RAP $(30 \%, 50 \%, 70 \%$ e $90 \%)$ e de cimento $(2 \%, 4 \%, 5 \%, 6 \%, 7 \%, 8 \%, 9 \%$ e $10 \%)$ na resistência das misturas. Os resultados de ISC demonstraram a viabilidade do uso da mistura solo-RAP sem cimento como sub-base de pavimento rodoviário e indicaram também a viabilidade do uso como base para baixos teores de cimento. Por fim, os ensaios de RCNC demonstraram que a utilização dos materiais dependerá do normativo considerado no projeto da mistura.

Palavras-chave: Material fresado; Agregado negro; Solo-cimento

\begin{abstract}
The use of waste in Road Engineering has been increased in several countries, including Brazil, due to the environmental and economic advantages associated to this activity. The present research aimed to evaluate mixtures of soil and RAP (Reclaimed Asphalt Pavement) to apply as road pavement layers, based on California Bearing Ratio (CBR) tests and Unconfined Compression Strenght tests (UCS). The study evaluated the influence of different RAP $(30 \%, 50 \%, 70 \%$ and $90 \%)$ and cement contents $(2 \%, 4 \%, 5 \%, 6 \%, 7 \%, 8 \%, 9 \%$ and $10 \%$ ) on the strenght of the mixtures. The CBR results demonstrated that it is possible to use soil-RAP mixture without cement as a sub-base for road pavement and also indicated the feasibility of using it as a base for low cement levels. Finally, UCS tests showed that the use of the materials will depend on the standards considered in the mixture design.
\end{abstract}


Keywords: Asphalt waste; Black aggregate; Soil-cement

\section{Introdução}

A destinação de resíduos de construção e demolição (RCD) é um dos grandes desafios da modernidade em todo o mundo. O crescente aproveitamento de resíduos na Engenharia Rodoviária vem ganhando força em vários países, em função dos ganhos ambientais e econômicos associados a esta atividade (Adhikari et al., 2018; Suddeepong et al., 2018; Ghanizadeh \& Rahrovan, 2019). No Brasil, a utilização de RAP (Reclaimed Asphalt Paviment), que é o material fresado de revestimentos asfálticos, é uma prática corrente na confecção de misturas asfálticas e camadas granulares recicladas de pavimentos rodoviários, em especial em rodovias concessionadas (Segundo et al., 2016; Gaspar et al., 2020; Unger Filho et al., 2020).

A dosagem de misturas de solo e RAP, entretanto, se mostra uma tarefa determinante na performance que $o$ material resultante terá, quando empregado como camada de sub-base ou base de pavimentos. Suebsuk et al. (2014), por exemplo, analisando a influência do teor de RAP no comportamento de misturas com solo arenoso laterítico tratadas com cimento, em termos dos parâmetros de compactação e resistência à compressão não confinada, verificaram que com o aumento no teor de RAP a umidade ótima da mistura tendeu a diminuir, até a proporção de $50 \%$ de RAP, indicando que este teor de resíduo, que correspondeu à presença de $3,5 \%$ de asfalto residual em massa envolvendo o agregado, foi definido como o ponto ótimo da mistura. Segundo os autores, à medida que o teor de asfalto aumentou, com o aumento do teor de RAP na mistura, a resistência à compressão não confinada diminuiu devido a uma redução no atrito entre as partículas sólidas (solo e RAP). Os autores testaram proporções de soloRAP de 80/20, 60/40, 50/50, 40/60 e 20/80, com adição de $1 \%, 3 \%$ e $5 \%$ de cimento em massa. Esta constatação é confirmada nos estudos realizados por Suebsuk et al. (2019).

Já Ochepo (2014), realizando um estudo laboratorial para avaliar a resistência de um solo laterítico, estabilizado com RAP e cinza de bagaço de cana, observou que a adição de RAP reduziu o teor de umidade ótima e aumentou a densidade seca máxima da amostra em comparação com o solo natural. Com base nos resultados laboratoriais obtidos e nos critérios de resistência adotados, os autores observaram que o tratamento com 6 a $8 \%$ em massa de bagaço de cana na mistura solo-RAP produziu resultados satisfatórios, para uso do material em camada de sub-base e com $10 \%$ habilitou o material para o uso como camada de base em vias de tráfego leve. O solo foi misturado com RAP na proporção $40 / 60$ e tratado com $0 \%, 2 \%, 4 \%, 6 \%, 8 \%$ e $10 \%$ de bagaço. O autor realizou ensaios de granulometria, compactação, Resistência à Compressão Não Confinada (RCNC) e Índice de Suporte Califórnia (ISC) nas misturas. Ghanizadeh et al. (2018) estudaram em laboratório a estabilização de misturas de RAP, solos granulares e cimento para aplicação em obras de reciclagem profunda, utilizando o revestimento deteriorado e base existentes, normalmente com auxílio de equipamento pesado conhecido como recicladora. Foram utilizados dois diferentes tipos de solos granulares. Os testes de compactação e RCNC foram realizados em diferentes proporções de RAP e solos granulares de 0/100, 20/80, 40/60 e 60/40. Foram adicionados quatro teores de cimento de $3 \%, 4 \%, 5 \%$ e $6 \%$ em massa, com as amostras submetidas a cura por 7 e 28 dias após a compactação. Os resultados mostraram que, para o mesmo teor de RAP, o acréscimo de $1 \%$ do teor de cimento correspondeu ao aumento médio da resistência a compressão não confinada de $390 \mathrm{kPa}$. Os resultados mostraram, ainda, que o teor ótimo de cimento para construção de camadas recicladas profundas está entre 3\% e 5\% para os materiais estudados.

Alhaji \& Alhassan (2018) avaliaram misturas de um solo argiloso do nordeste da Nigéria e RAP, com teores entre $0 \%$ a $100 \%$, com variações progressivas de $10 \%$ em $10 \%$ de RAP entre fases experimentais. Dentre outros parâmetros, os autores avaliaram a resistência das misturas compactadas. Um teste de extração indicou o teor de asfalto residual presente no RAP de 5,99\% em massa. Os resultados da compactação das misturas na energia modificada mostraram que a densidade seca máxima aumentou com $30 \%$ de RAP, em relação $0 \%$ de RAP, e caiu com $100 \%$ de RAP. O teor ótimo de umidade, no entanto, diminuiu de $13,7 \%$ com $0 \%$ RAP, para $8,8 \%$ entre $40 \%$ e $60 \%$ de RAP, após o qual o valor aumentou para $9,5 \%$ a $90 \%$ de RAP. O ISC alcançou valor máximo de $35 \%$ com $30 \%$ de RAP, após o qual o valor caiu para 5\% com $100 \%$ de RAP. Os autores concluíram que $30 \%$ de RAP se mostrou a mistura ideal, oferecendo resistência compatível para uso em camada de base de estradas com tráfego leve na Nigéria. Portanto, concluem os autores, o teor de ligante asfáltico residual de 3,07\%, obtido para esta mistura, pode ser adotado como ponto de fixação para as misturas estudadas.

Fedrigo et al. (2015) estudaram a influência do teor de RAP $(20 \%, 50 \%$ e $70 \%)$, da energia de compactação (intermediária e modificada) e do tempo de cura (3, 7 e 14 dias) na RCNC de misturas com Brita Graduada Simples (BGS). Os resultados indicaram o tempo de cura de 7 dias como adequado, com a maior energia de compactação promovendo aumento da RCNC, enquanto que o aumento do teor de RAP para $70 \%$ promoveu redução deste parâmetro. 
Acompanhando essa tendência, o presente estudo tem como objetivo avaliar misturas solo-RAP e solo-RAP com cimento, para utilização como camada de sub-base e base de pavimentos rodoviários, de acordo com as exigências de diferentes órgãos rodoviários brasileiros.

\section{Material e Métodos}

Esta pesquisa iniciou-se pela caracterização dos materiais, seguida pela execução dos ensaios de compactação e Índice de Suporte Califórnia (ISC) ou CBR (California Bearing Ratio) e, por fim, pelos ensaios de resistência à compressão não confinada, os quais serão detalhados ao longo deste item. Para os ensaios lançou-se mão da utilização de normas específicas para aplicações em obras rodoviárias, além de normatização da Associação Brasileira de Normas Técnicas, relativas às aplicações práticas deste estudo.

\subsection{Caracterização dos Materiais}

\subsubsection{Caracterização Geotécnica e Química do Solo}

O solo estudado é um Latossolo amarelo distrocoeso, que ocorre na maior parte do Recôncavo da Bahia, proveniente do campo experimental da Universidade Federal do Recôncavo da Bahia (UFRB). Segundo Rodrigues et al. (2009), este solo é bastante profundo, sobrejacente ao material sedimentar e ocupa áreas de relevo plano e suave ondulado dos tabuleiros.

Os Latossolos, classificados como lateríticos, são constituídos por material mineral em avançado estágio de intemperização e possuem baixa capacidade de troca de cátions da fração argila, com predominância do argilomineral caulinita (EMBRAPA, 2018).

$\mathrm{Na}$ caracterização geotécnica foram realizados os ensaios de granulometria por peneiramento, segundo a NBR 7181 (ABNT, 2018), limite de liquidez, de acordo com a NBR 6459 (ABNT, 2016a), limite de plasticidade, conforme a NBR 7180 (ABNT, 2016b) e densidade real, segundo a DNER ME 93 (DNER, 1994a).

Na caracterização química, para identificação do argilomineral presente na fração fina, foi realizada uma avaliação para os seguintes atributos químicos: $\mathrm{pH}$ em água e em KCl (relação solo:solução de 1:2,5); cátions trocáveis, por titulação após extração com solução de 1 mol KCl; por fotometria de chama, após extração com Mehlich-1; $\mathrm{H}+\mathrm{Al}$ extraídos com acetato de cálcio $0,5 \mathrm{~mol} / \mathrm{L}$ a pH 7,0 e determinado com $\mathrm{NaOH} 0,025 \mathrm{~mol} / \mathrm{L}$. Baseado nestes dados e no teor de argila do solo, foram calculadas a capacidade de troca de cátions (CTC), pela soma do resultado de bases trocáveis $\left(\mathrm{Ca}^{2+}+\mathrm{Mg}^{2+}+\mathrm{Na}^{+}+\mathrm{K}^{+}\right)$ e acidez $(\mathrm{H}+\mathrm{Al})$ e, a atividade da argila $(\mathrm{T})$, referente à capacidade de troca de cátions correspondente à fração argila, calculada pela Equação 1, além de calcular saturação por base (V) (EMBRAPA, 2018). Os teores $\mathrm{de}^{\mathrm{SiO}_{2}}, \mathrm{Al}_{2} \mathrm{O}_{3} \mathrm{e}$ $\mathrm{Fe}_{2} \mathrm{O}_{3}$, presentes na fração de argila do solo, também foram avaliados a fim de se determinar as relações moleculares Ki e $\mathrm{Kr}$ (Equações 2 e 3, respectivamente), as quais indicam a presença de argilominerais (EMBRAPA, 2018).

$$
\begin{gathered}
T=\frac{C T C \cdot 100}{\text { teor de argila }(\%)} \\
\mathrm{Ki}=1,70 \cdot \frac{\mathrm{SiO}_{2}}{\mathrm{Al}_{2} \mathrm{O}_{3}} \\
\mathrm{Kr}=1,70 \cdot \frac{\mathrm{SiO}_{2}}{\mathrm{Al}_{2} \mathrm{O}_{3}+\mathrm{Fe}_{2} \mathrm{O}_{3} \cdot 0,6375}
\end{gathered}
$$

\subsubsection{Caracterização do Solo pela Metodologia MCT}

Os ensaios de compactação do solo em miniatura de amostras (Mini-MCV) e o de perda de massa por imersão foram realizados, para classificação pela metodologia MCT. O primeiro consistiu na compactação de corpos de prova em miniatura $(\varnothing=50 \mathrm{~mm})$, com energias variáveis por meio de golpes sucessivos no compactador Mini-MCV. A altura do corpo de prova foi verificada com a utilização de extensômetro, interrompendo-se o processo quando atingisse uma das três condições: a) diferença inferior a 2 $\mathrm{mm}$ entre a leitura após $4 \mathrm{n}$ golpes e aquela após $\mathrm{n}$ golpes; b) intensa exsudação de água no topo e na base do corpode-prova; c) aplicação de 256 golpes.

No ensaio de perda de massa por imersão, os corpos de prova compactados foram extrudados em $10 \mathrm{~mm}$ para fora do molde cilíndrico. Em seguida, o conjunto foi colocado em posição horizontal e imerso em água, de forma a garantir uma lâmina d'água não inferior a $1 \mathrm{~cm}$ acima da superfície externa do molde. Foram colocadas cápsulas para coleta do solo, no caso de desprendimento de porções dos corpos de prova. Os conjuntos ficaram imersos por cerca de 20 horas. Os ensaios de Mini-MCV e perda de massa por imersão foram realizados conforme os procedimentos prescritos nas normas DNER CLA 259 (DNER, 1996), DNER ME 258 (DNER, 1994b) e DNER ME 256 (DNER, 1994c).

\subsubsection{Caracterização do RAP}

O RAP foi proveniente dos serviços de fresagem do revestimento das rodovias BR 116/BA e BR 324/BA, de mesma constituição e idade, coletado durante as obras de restauração de segmentos das duas rodovias (Figura 
1), cujo teor de ligante asfáltico residual médio foi de $5,5 \%$ em massa, determinado pelo método de extração por centrifugação segundo a DNER ME 53 (DNER, 1994d).

Devido à heterogeneidade da constituição deste material, o mesmo foi fracionado utilizando as peneiras 3/4" (19,1 mm) e \#4 (4,8 mm), sendo dividido em três porções (grosso, médio e fino), e determinando a respectiva porcentagem. A parcela grossa, a qual apresentou a menor proporção e quantidade expressiva de grumos, foi substituída
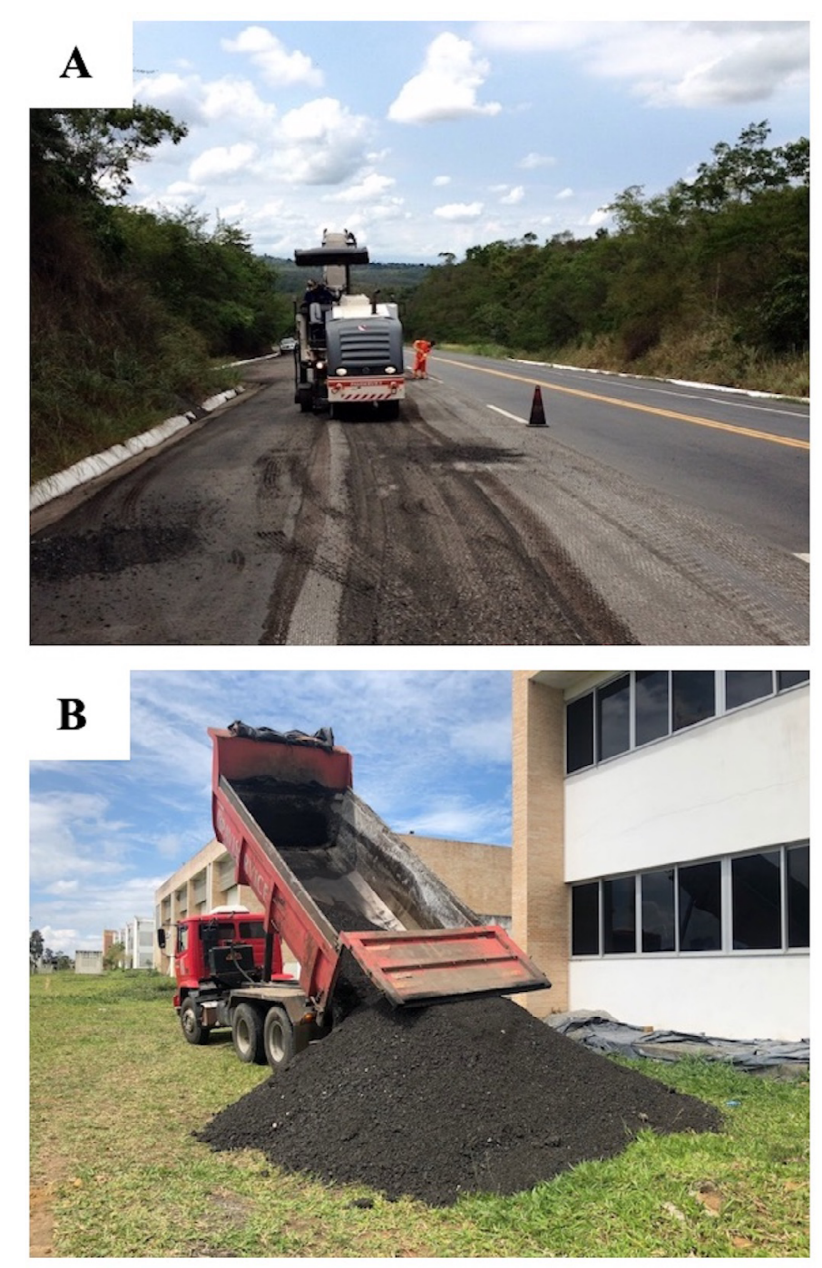

Figura 1 Geração e descarga do RAP: (A) operação de fresagem; (B) material fresado (RAP).

por fração média equivalente, obtendo-se assim um RAP mais homogêneo, constituído de $38,1 \%$ de fração média e $61,9 \%$ da fração fina. Com esse material, realizou-se ensaio de análise granulométrica por peneiramento segundo a NBR 7181 (ABNT, 2018) e densidade real segundo a DNER ME 81 (DNER, 1998).

\subsubsection{Misturas Solo-RAP}

Os experimentos com as misturas de solo, RAP e cimento foram divididos em cinco etapas, conforme apresentado na Tabela 1.

Foram testados o solo e RAP puros e misturas solo-RAP com percentuais de $30 \%, 50 \%, 70 \%$ e $90 \%$ de RAP, como forma de buscar avaliar a maior variação possível de misturas. $\mathrm{O}$ cimento utilizado nas misturas foi o CPII-Z, cujas quantidades foram calculadas em relação à massa total da mistura seca, da mesma forma que o RAP. As misturas sem cimento foram submetidas a ensaios de granulometria por peneiramento, conforme a NBR 7181 (ABNT, 2018) e todas as misturas foram submetidas a ensaios de compactação.

\subsection{Ensaios de Compactação}

Estes ensaios foram realizados em amostras não trabalhadas de todos os materiais e misturas do presente estudo, para determinação das curvas de compactação e, consequente, obtenção da umidade ótima e massa específica seca máxima de cada amostra $\left(\mathrm{w}_{\text {ot }}\right.$ e $\left.\rho_{\text {dmáx }}\right)$.

As curvas de compactação do solo, RAP, misturas sem cimento e misturas com $2 \%, 4 \%$ e $5 \%$ de cimento foram determinadas com cinco pontos na Energia Modificada, com uso do molde de compactação Proctor em miniatura para corpos de prova de diâmetro de $50 \mathrm{~mm}$ e altura de $100 \mathrm{~mm}$. Entretanto, utilizou-se a Energia Normal, com o mesmo molde, para as misturas contendo entre $6 \% \mathrm{e}$ 10\% de cimento, segundo DNIT ES 143 (DNIT, 2010a). Para verificar a possibilidade de utilização do molde em miniatura, para obtenção dos parâmetros ótimos de compactação das misturas, foi feita uma comparação com curvas produzidas no molde grande convencionalmente adotado no método de ensaio DNIT 164 (DNIT, 2013). A massa específica seca máxima obtida com o equipamento em miniatura, para as misturas com no máximo $4 \%$ de cimento, foram relacionadas com aquelas obtidas nas compactações realizadas para os ensaios de ISC, sendo definida a Relação entre os Parâmetros de Compactação (RPC) como a razão percentual entre as massas específicas secas máximas da amostra pequena e da grande, para cada material ensaiado.

Cabe destacar que a compactação das amostras no molde em miniatura, utilizadas em todo o programa experimental, foi controlada de forma que só foram utilizados Corpos de Prova (CP) com Graus de Compactação (GC) de $100 \% \pm 2 \%$ e umidade com variação de $\pm 0,5 \%$, em relação a seus respectivos parâmetros ótimos de compactação. As amostras que não atenderam a esse critério foram descartadas. 
Para a compactação de cada CP no molde em miniatura, separou-se $500 \mathrm{~g}$ de material para cada teor de umidade. Importante destacar que as amostras de solo e soloRAP, preparadas para compactação no molde em miniatura após serem umedecidas (Figura 2A), permaneceram durante $12 \mathrm{~h}$ acondicionadas dentro de sacos plásticos (Figura $2 \mathrm{~B}$ ), para uniformização da umidade no material e evitar a perda ou ganho de umidade. Entretanto, para as amostras com $2 \%$ e $4 \%$ de cimento, compactadas no molde convencional e no molde Proctor em miniatura, além das amostras com $5 \%$ de cimento, compactadas no molde em miniatura, seguiu-se as recomendações da norma DNIT ES 142 (DNIT, 2010b), submetendo as misturas a um período de cura de 72 horas, acondicionadas em recipientes plásticos, antes da compactação (Figura 2B). Por fim, as amostras contendo entre $6 \%$ e $10 \%$ de cimento foram compactadas, logo após a homogeneização.

No ensaio de compactação, a umidade de cada ponto das curvas foi determinada em estufa a $105^{\circ} \mathrm{C}$ e por, no mínimo, $24 \mathrm{~h}$. Após a compactação, os corpos de prova produzidos foram desmoldados, pesados e tiveram suas respectivas alturas e diâmetros determinados. Com estes dados foi possível calcular a massa específica aparente seca de cada ponto ensaiado.

\subsection{Ensaios de ISC}

Os ensaios de ISC foram realizados nas amostras solo-RAP sem cimento e nas amostras com 50\% e 70\% de RAP com $2 \%$ e $4 \%$ de cimento, conforme Tabela 1 . Nas amostras sem cimento, os ensaios foram realizados de acordo com a norma DNIT ME 172 (DNIT, 2016). Os corpos de prova após compactados foram colocados em imersão, com leituras de expansão a cada $24 \mathrm{~h}$, por 4 $\operatorname{dias}(96 \mathrm{~h})$.

As misturas com cimento, entretanto, após a compactação foram imediatamente submetidas ao ensaio de ISC, sem imersão prévia, ou seja, na umidade de compactação conforme a DER-BA ES-P-06/01 (DER$\mathrm{BA}, 2001)$. Após serem rompidos na prensa, os corpos de prova foram inseridos no tanque para leituras diárias de expansão durante 4 dias.

\subsection{Ensaios de compressão não confinada (RCNC)}

As amostras para os ensaios de Resistência à Compressão Não Confinada (RCNC) foram preparadas, utilizando-se o molde e a mesma metodologia de preparo do ensaio de compactação. Para cada mistura e teor de umidade ótima (determinado no ensaio de compactação), preparou-se três CPs que foram submetidos ao ensaio de RCNC.

Para as misturas sem cimento, logo após a compactação, os CPs foram envolvidos em filme plástico para evitar a variação de umidade, sendo após $24 \mathrm{~h}$ submetidos ao ensaio de RCNC.

Para as misturas com cimento, adotou-se as normas DNER ME 202 (DNER, 1994e) e NBR 12253 (ABNT, 2012), as quais prescrevem os procedimentos e condições requeridas para preparação e dosagem de misturas solocimento utilizando corpos de prova cilíndricos. Após a compactação das amostras com cimento, os CPs permaneceram por sete dias dentro de um dessecador (Figura 3A), devidamente vedado, com controle de umidade

Tabela 1 Etapas, dosagens e ensaios realizados.

\begin{tabular}{|c|c|}
\hline Mistura & Ensaios \\
\hline Solo puro & $\begin{array}{c}\text { ISC } \\
\text { RCNC }\end{array}$ \\
\hline $\begin{array}{c}\text { Solo } \\
+ \\
(30 \%, 50 \%, 70 \% \text { e } 90 \%) \text { de RAP }\end{array}$ & $\begin{array}{c}\text { ISC } \\
\text { RCNC }\end{array}$ \\
\hline RAP puro & $\begin{array}{c}\text { ISC } \\
\text { RCNC }\end{array}$ \\
\hline $\begin{array}{c}\text { Solo } \\
+ \\
(50 \% \text { e } 70 \%) \text { de RAP } \\
+ \\
(2 \% \text { e } 4 \%) \text { de Cimento }\end{array}$ & $\begin{array}{c}\text { ISC } \\
\text { RCNC }\end{array}$ \\
\hline $\begin{array}{c}\text { Solo } \\
+ \\
50 \% \text { de RAP } \\
+ \\
\text { Cimento } \\
(5 \%, 6 \%, 7 \%, 8 \%, 9 \% \text { e } 10 \%)\end{array}$ & RCNC \\
\hline
\end{tabular}



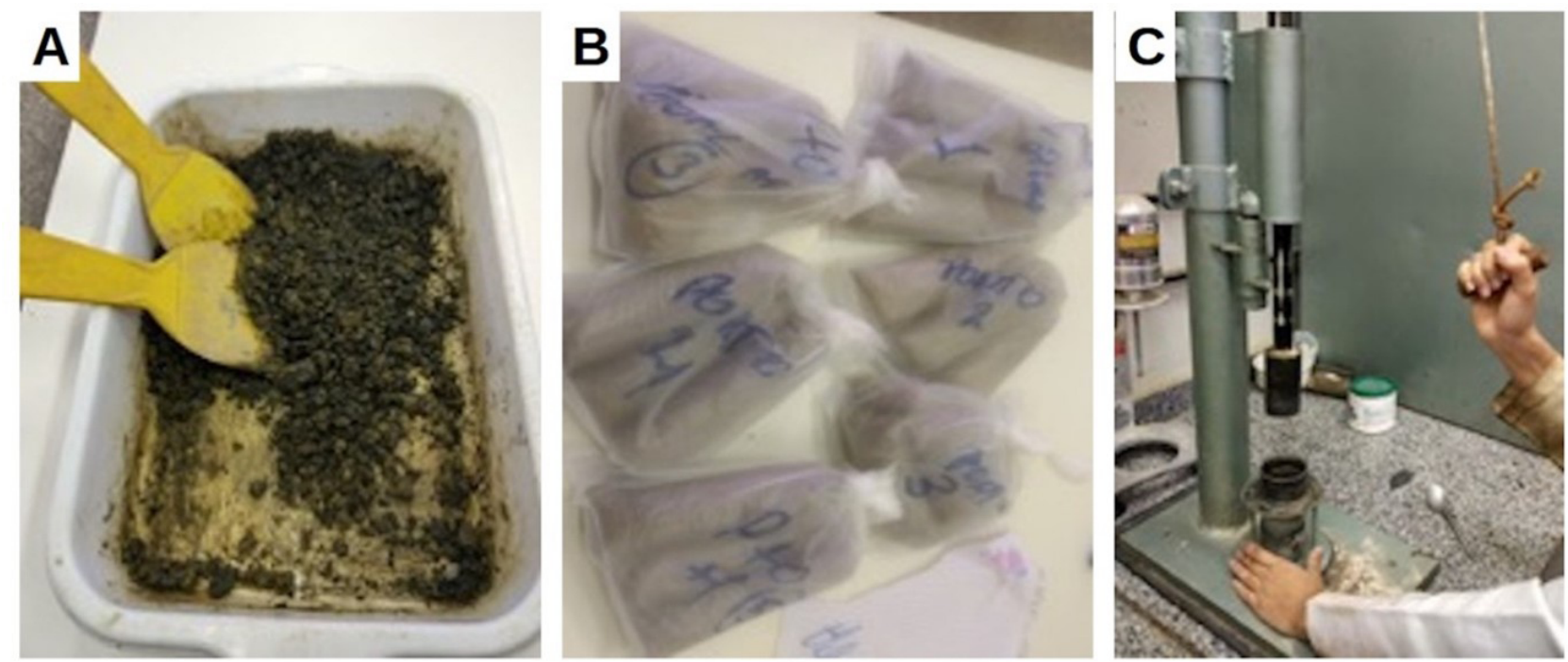

Figura 2 Etapas de umedecimento $(A)$ e acondicionamento $(B)$ da preparação das amostras e compactação no molde Proctor em miniatura $(C)$.
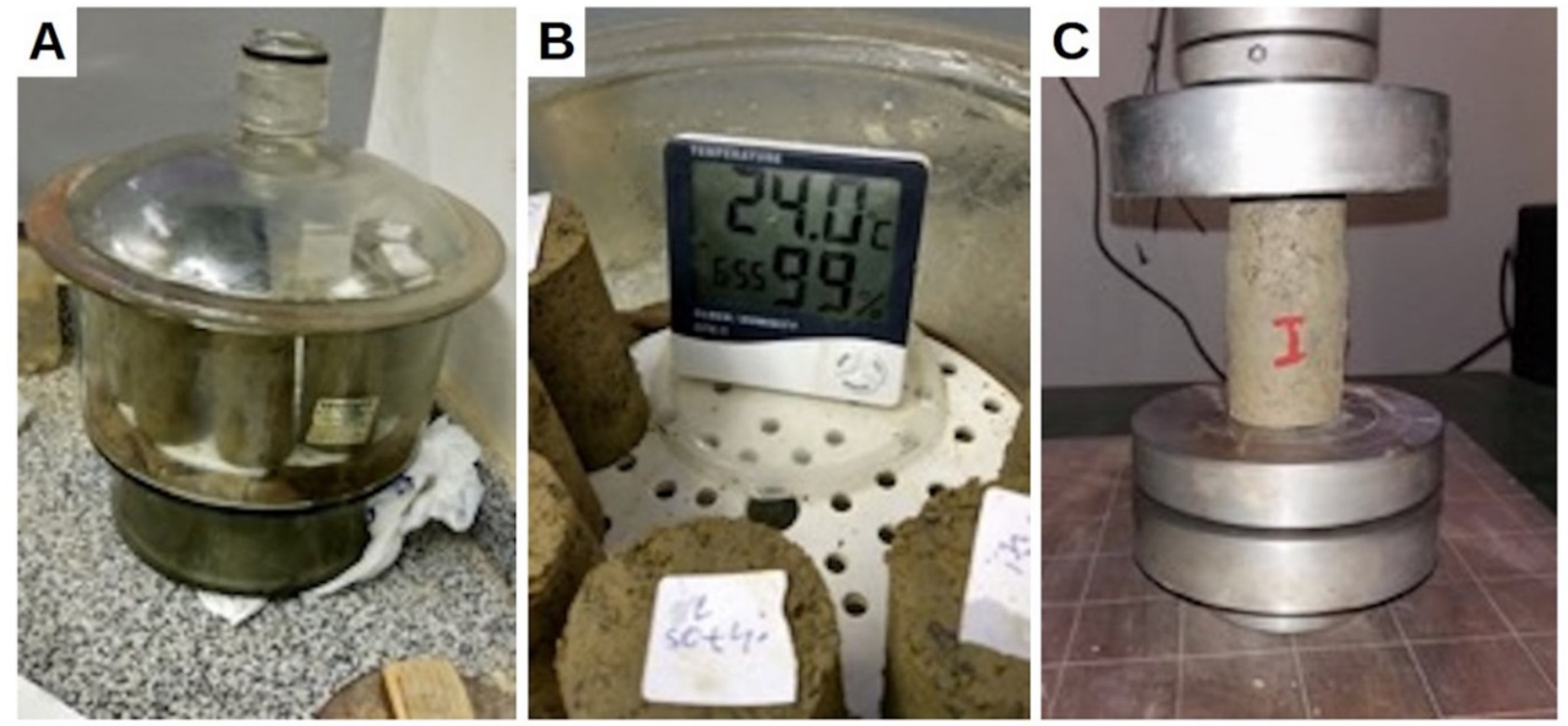

Figura 3 Etapas de preparação (A) e (B) e do ensaio de RCNC (C).

relativa do ar $(\geq 95 \%)$ e temperatura $\left(23^{\circ} \mathrm{C} \pm 2\right)$, em processo de cura (Figura 3B), sendo depois submetidos ao ensaio de RCNC (Figura 3C).

Os ensaios de resistência foram realizados na Máquina Universal Times Group, com velocidade de 1 $\mathrm{mm} / \mathrm{min}$ (Figura $3 \mathrm{C}$ ), como recomenda a norma DNER ME 201 (DNER, 1994f).
Importante destacar que, durante a campanha experimental, percebeu-se a impossibilidade de execução do ensaio de compressão não confinada em amostras de mistura de solo com $90 \%$ de RAP e no RAP puro, em função da baixa coesão entre as partículas, propiciada pelas particularidades do material fresado, que possui $38,1 \%$ retido entre as peneiras com abertura $19,1 \mathrm{~mm}$ e $4,8 \mathrm{~mm}$, ou seja, equivalente a 
pedregulho e, aproximadamente, $61,9 \%$ de partículas com dimensões equivalentes a areias grossa e média, resultando na desagregação do material após desmoldagem dos CPs.

Posteriormente à obtenção dos resultados, calculouse as médias, desvios padrões e coeficientes de variação (CV) dos resultados de resistência à compressão não confinada obtidos em triplicata.

\section{Resultados e Discussões}

\subsection{Caracterização dos Materiais}

Os resultados dos ensaios de caracterização química e geotécnica do solo estão apresentados nas Tabelas 2 e 3 , respectivamente, junto com a massa específica do RAP (Tabela 2).

Na Tabela 2, os índices Ki e Kr refletem o grau de intemperização do solo e indicam o argilomineral presente no mesmo. Quando Ki e Kr são superiores a 0,75, existe uma predominância de argilominerais do grupo caulinita (1:1). A presença de montmorilonilta (2:1) fica definida quando se tem Ki superior a 2,2, e alta capacidade de troca de cátions (> $17 \mathrm{cmol} / \mathrm{kg})$ (EMBRAPA, 2018).

Como pode ser observado, o solo apresenta mineralogia caulinítica e os resultados obtidos para $\mathrm{V}$ e CTC confirmam sua classificação como Latossolo, uma vez que possui baixa saturação por bases $(\mathrm{V}<50 \%)$, capacidade de troca de cátions inferior a $17 \mathrm{cmol} / \mathrm{kg}$, e, consequentemente, uma atividade baixa da fração argila, ocasionada pelo avançado processo de intemperização.

Na Tabela 3, observa-se que a massa específica dos sólidos do RAP é maior que o valor encontrado para o solo estudado. Valores maiores indicam a predominância de finos em se tratando de solos. Entretanto, como a massa específica dos sólidos representa a média aritmética dos minerais que compõem o material, no caso do RAP a predominância de materiais britados, cujas massas específicas individuais são elevadas, com pouca influência do ligante asfáltico residual presente, justifica o seu valor elevado de massa específica dos sólidos.

Ainda da Tabela 2, o índice de plasticidade igual a $10 \%$ (média plasticidade) e a massa específica dos sólidos indicam predominância de comportamento arenoso. Embora pela classificação TRB (A - 4), o solo tenha sido enquadrado como siltoso com pequena quantidade de material grosso e argila, esta classificação não se aplica por não evidenciar as características específicas de tal solo laterítico. Para este caso, utiliza-se a metodologia MCT, apropriada a solos lateríticos. Desta forma, de acordo com os resultados de Mini-MCV e perda de massa por imersão, o solo estudado apresenta comportamento compatível com areias argilosas bem graduadas, que possuem argila de natureza laterítica em sua composição sendo, portanto, classificado como LG'. Tais solos, ainda de acordo com a metodologia MCT, possuem baixa expansão, média contração, podendo ser empregados como revestimento primário, proteção à erosão, base de pavimento, subleito compactado e reforço de subleito compactado (NOGAMI \& VILLIBOR, 1995).

\subsection{Granulometria}

As granulometrias do solo, RAP e das misturas avaliadas estão apresentadas na Tabela 4. Com as granulometrias apresentadas na Tabela 4, é possível avaliar cada material e mistura com base nas quatro normas de dosagem abordadas no presente estudo. Assim, de acordo com a norma DNIT ES 141 (DNIT, 2010c), apenas as misturas com $50 \%$ e $70 \%$ de RAP se enquadram, granulometricamente, nas faixas $\mathrm{F}$ e E, respectivamente, para utilização como camada de base granular de pavimentos rodoviários. Pela norma DNIT ES 142 (DNIT, 2010b),

Tabela 2 Caracterização química do Solo.

\begin{tabular}{cc}
\hline Propriedade & Valor \\
\hline $\mathrm{V}(\%)$ & 40,00 \\
$\mathrm{Al}_{2} \mathrm{O}_{3}(\%)$ & 8,94 \\
$\mathrm{SiO}_{2}(\%)$ & 12,43 \\
$\mathrm{Fe}_{2} \mathrm{O}_{3}(\%)$ & 3,47 \\
$\mathrm{CTC}(\mathrm{cmol} / \mathrm{kg})$ & 3,10 \\
$\mathrm{Ki}$ & 2,36 \\
$\mathrm{Kr}$ & 1,90 \\
$\mathrm{~T}(\mathrm{cmol} / \mathrm{kg})$ & 8,09 \\
\hline
\end{tabular}


Tabela 3 Caracterização dos materiais.

\begin{tabular}{cc}
\hline Propriedades do solo & Valor \\
\hline Limite de liquidez $-\mathrm{w}_{\mathrm{L}}(\%)$ & 31 \\
Limite de plasticidade $-\mathrm{w}_{\mathrm{P}}(\%)$ & 21 \\
Índice de plasticidade $-\mathrm{PP}(\%)$ & 10 \\
Massa específica dos sólidos $-\rho_{\mathrm{S}}\left(\mathrm{g} / \mathrm{cm}^{3}\right)$ & 2,59 \\
Classificação TRB & $\mathrm{A}-4$ \\
IG & 4 \\
Classificação MCT & LG' \\
Cor & Amarelo \\
\hline Massa específica do RAP $\left(\mathrm{g} / \mathrm{cm}^{3}\right)$ & 2,73 \\
\hline
\end{tabular}

Tabela 4 Granulometrias dos materiais e misturas utilizados no estudo.

\begin{tabular}{|c|c|c|c|c|c|c|}
\hline \multirow[b]{2}{*}{ Peneira } & \multirow{2}{*}{$\begin{array}{c}\text { RAP } \\
\% \text { que passa }\end{array}$} & \multirow{2}{*}{$\begin{array}{c}\text { Solo } \\
\% \text { que passa }\end{array}$} & \multicolumn{4}{|c|}{ Misturas solo-RAP } \\
\hline & & & $\begin{array}{l}30 \% \text { de RAP } \\
\% \text { que passa }\end{array}$ & $\begin{array}{l}50 \% \text { de RAP } \\
\% \text { que passa }\end{array}$ & $\begin{array}{l}70 \% \text { de RAP } \\
\% \text { que passa }\end{array}$ & $\begin{array}{l}90 \% \text { de RAP } \\
\% \text { que passa }\end{array}$ \\
\hline $2 "$ & 100,0 & 100,0 & 100,0 & 100,0 & 100,0 & 100,0 \\
\hline $1 "$ & 100,0 & 100,0 & 100,0 & 100,0 & 100,0 & 100,0 \\
\hline $3 / 8 "$ & 92,1 & 100,0 & 97,6 & 93,5 & 94,4 & 92,8 \\
\hline$\# 4$ & 65,8 & 100,0 & 89,7 & 81,3 & 76,0 & 69,2 \\
\hline$\# 10$ & 41,9 & 99,7 & 82,3 & 71,3 & 59,2 & 47,7 \\
\hline$\# 40$ & 6,9 & 79,6 & 57,8 & 42,5 & 28,7 & 14,1 \\
\hline \#200 & 0,4 & 54,4 & 38,2 & 24,1 & 16,6 & 5,8 \\
\hline
\end{tabular}

apenas a mistura com $70 \%$ de RAP se enquadra (faixa D), para misturas melhoradas com cimento. Considerando a norma DNIT ES 143 (DNIT, 2010a), entretanto, apenas a mistura com $90 \%$ de RAP não se mostra apta para uso como base de solo-cimento. Por fim, conforme a norma DER-PR ES-P 11/05 (DER-PR, 2005), as quatro misturas com RAP se mostram granulometricamente aptas, para uso como camada de sub-base e base com adição de cimento.

\subsection{Compactação}

Os parâmetros de compactação ( $\mathrm{w}_{\mathrm{ot}}$ e $\left.\rho_{\text {dmáx }}\right)$ do solo, RAP e misturas estão apresentados na Tabela 5 , junto com os valores da relação entre os parâmetros de compactação (RPC), usados para verificação da compactação no molde em miniatura, os quais ficaram compreendidos entre $95 \% \mathrm{e}$ $105 \%$. Desse modo foi considerada adequada a compactação no molde miniatura no presente estudo, mesmo o material apresentando partículas maiores que $2 \mathrm{~mm}$. Somente os CPs com GC de $100 \% \pm 2 \%$ e umidade com variação de \pm
$0,5 \%$, em relação a seus respectivos parâmetros ótimos de compactação, foram utilizados nos ensaios de resistência.

A massa específica aparente seca máxima alcançada pelo RAP na energia modificada, de $1,70 \mathrm{~g} / \mathrm{cm}^{3}$, é $10,5 \%$ menor que a alcançada pelo solo $\left(1,90 \mathrm{~g} / \mathrm{cm}^{3}\right)$, coerente com a massa específica dos sólidos de ambos, pois como o RAP possui massa específica dos sólidos equivalente à de solos com predominância de finos, a sua massa específica aparente seca máxima deve ser inferior à do solo. Isto pode ser explicado pela presença de ligante asfáltico residual no RAP, o qual sendo essencialmente hidrofóbico, interfere na capacidade do RAP de atingir uma maior densificação.

O aumento do teor de RAP nas misturas proporcionou elevação da massa específica seca máxima até os teores de $50 \%$ e $70 \%$, com valores de $\rho_{\text {dmáx }}$ de $2,01 \mathrm{~g} / \mathrm{cm}^{3}$ e 2,04 $\mathrm{g} / \mathrm{cm}^{3}$, respectivamente, com queda desse parâmetro para $90 \%$ de RAP.

Isto indica que o teor ótimo de RAP, para as misturas testadas, situa-se entre esses valores $(50 \%$ e $70 \%)$, conforme evidenciado por Suebsuk et al. (2014) e Ghanizadeh et al. (2018). 
Tabela 5 Parâmetros ótimos de compactação.

\begin{tabular}{|c|c|c|c|c|}
\hline$\%$ de RAP & $\%$ de cimento & $w_{o t}(\%)$ & $\rho_{d \max }\left(\mathbf{g} / \mathbf{c m}^{3}\right)$ & RPC (\%) \\
\hline 0 (Solo) & 0 & 13,14 & 1,90 & 100 \\
\hline 100 (RAP) & 0 & 7,14 & 1,70 & 96 \\
\hline 30 & 0 & 10,83 & 1,99 & 102 \\
\hline 50 & 0 & 9,08 & 2,01 & 103 \\
\hline 70 & 0 & 8,29 & 2,04 & 103 \\
\hline 90 & 0 & 7,63 & 1,87 & 95 \\
\hline 50 & 2 & 9,74 & 1,99 & 101 \\
\hline 50 & 4 & 11,49 & 1,92 & 103 \\
\hline 70 & 2 & 7,59 & 1,96 & 99 \\
\hline 70 & 4 & 8,99 & 1,99 & 104 \\
\hline 50 & 5 & 12,06 & 1,92 & - \\
\hline 50 & 6 & 12,13 & 1,94 & - \\
\hline 50 & 7 & 11,62 & 1,97 & - \\
\hline 50 & 8 & 12,05 & 1,95 & - \\
\hline 50 & 9 & 10,13 & 1,98 & - \\
\hline 50 & 10 & 10,06 & 1,99 & - \\
\hline
\end{tabular}

Entretanto, o teor de umidade ótima do RAP é coerente com a sua granulometria grossa, sendo menor que o valor encontrado para o solo, cuja granulometria é mais fina que a do RAP (ver Tabela 4). Isto também explica os valores de umidade ótima inferiores ao do solo, para todas as misturas.

Observa-se que a adição de cimento, em geral, reduz a densidade seca máxima da mistura, quando comparado aos resultados das misturas solo-RAP, mas aumenta se comparado ao solo isoladamente.

Com a adição do cimento às misturas, o teor de umidade ótima aumentou para a maioria, quando comparado à mistura solo-RAP, visto que o cimento possui granulometria fina e, portanto, requer maior quantidade de água para homogeneização, devido à maior superfície específica. Ao comparar com o solo, a umidade ótima foi inferior, devido à presença do RAP que tem predominância de grossos.

De maneira geral, comparando-se com a mistura solo-RAP, a adição de cimento não trouxe vantagens, pois aumentou a umidade ótima e, portanto, a quantidade de água para promover a mistura, e reduziu a massa específica seca máxima, reduzindo a densificação.

\subsection{Avaliação do Solo-RAP sem Cimento}

Os resultados dos ensaios de ISC, expansão e valores médios de RCNC ( $\sigma r$ ), para os três CPs, com os respectivos coeficientes de variação (CV) do solo, RAP e misturas de solo com $30 \%, 50 \%$ e $70 \%$ de RAP estão apresentados na Tabela 6.

Na Tabela 6, observa-se que a adição do RAP no solo estudado promove uma queda no ISC das misturas, excetuando-se para $70 \%$ e $90 \%$ de adição, que se mostram habilitadas para uso como camada de sub-base, uma vez que a exigência da norma DNIT ES 139 (DNIT, 2010d) é de ISC $\geq 20 \%$.

É interessante observar o resultado da mistura com $90 \%$ de RAP, onde se tem o melhor comportamento quanto ao ISC, evidenciando o efeito do confinamento deste ensaio, ratificado pela falta de coesão comprovada no resultado de RCNC.

Para base granular de pavimento rodoviário, os valores de ISC mínimos exigidos pela norma DNIT ES 142 (DNIT, 2010b), ou seja, $60 \%$ para tráfego leve ( $\mathrm{N} \leq 5$ x $\left.10^{6}\right)$ e $80 \%$ para tráfego pesado $\left(\mathrm{N}>5 \times 10^{6}\right)$, não foram atendidos por nenhum dos materiais ou misturas testados.

Os resultados médios de RCNC encontrados, embora com elevados coeficientes de variação principalmente para o solo e a mistura com $70 \%$ de RAP (CV $>10 \%)$, mostram que esta mistura apresentou a maior resistência. 
Tabela 6 ISC, expansão e RCNC dos materiais e misturas sem cimento na Energia Modificada.

\begin{tabular}{|c|c|c|c|c|}
\hline Materiais & $\begin{array}{l}\text { ISC } \\
(\%)\end{array}$ & $\begin{array}{c}\text { Expansão } \\
(\%)\end{array}$ & $\begin{array}{c}\sigma_{\mathrm{r}} \\
(\mathrm{MPa})\end{array}$ & $\begin{array}{c}\operatorname{CV}\left(\sigma_{r}\right) \\
(\%)\end{array}$ \\
\hline Solo & 20,20 & 0,05 & 0,37 & 16,21 \\
\hline RAP & 15,00 & 0 & - & - \\
\hline $30 \%$ de RAP & 19,50 & 0 & 0,07 & 0 \\
\hline $50 \%$ de RAP & 17,90 & 0 & 0,11 & 9,09 \\
\hline $70 \%$ de RAP & 20,40 & 0 & 0,18 & 11,11 \\
\hline $90 \%$ de RAP & 26,80 & 0 & - & - \\
\hline
\end{tabular}

Os resultados também mostram que houve aumento da RCNC, quanto maior o percentual de RAP, ocorrendo aumento considerável, quando a adição passou de $30 \%$ para $50 \%$ de RAP. Apesar disso, todas as misturas mostraram redução significativa da resistência, quando comparadas ao comportamento do solo isoladamente, sendo que a mistura com $70 \%$ de RAP alcançou apenas $49 \%$ da resistência do solo, ou seja, o resíduo, nos teores testados, não foi capaz de acrescentar resistência não confinada ao solo, diferentemente dos ensaios de ISC, evidenciando a necessidade de adicionar cimento à mistura.

\subsection{Avaliação do solo-RAP como Mistura Melhorada ou Tratada com Cimento}

Após a avaliação dos materiais e misturas soloRAP sem cimento, a Tabela 7 mostra os resultados de ISC, expansão e valores médios de RCNC $\left(\sigma_{r}\right)$, com os respectivos coeficientes de variação $(\mathrm{CV})$, para o solo, RAP e as misturas contendo $50 \%$ e $70 \%$ de RAP, com adição de $2 \%$ e $4 \%$, além dos resultados de RCNC dos mesmos percentuais de RAP e cimento.

Os resultados de ISC e RCNC médios obtidos para as amostras com adição de cimento, mostrados na Tabela 6 , indicam que de acordo com a norma DNIT ES 142 (DNIT, 2010b) as misturas contendo $50 \%$ e $70 \%$ de RAP, com $2 \%$ e $4 \%$ de cimento, apresentaram valores de ISC superiores a $80 \%$, credenciando essas misturas para utilização em camada de base de pavimento com tráfego pesado $(\mathrm{N}>$ $5 \times 10^{6}$ ).

Contudo, entre estas misturas aquela composta por $50 \%$ de RAP e $4 \%$ de cimento foi a que apresentou o maior valor de ISC, com queda de resistência ao aumentar o teor de RAP para 70\%, conforme observado por Fedrigo et. al. (2015), corroborando com a exigência da DEINFRA-SC ES-P09/16 (DEINFRA-SC, 2016) que estabelece 50\% como o teor máximo de RAP para misturas recicladas para base. É interessante ainda observar que com o aumento do teor de cimento de 2 para $4 \%$ na mistura com $70 \%$ de RAP, o valor de ISC praticamente não variou. Entretanto, nenhuma mistura apresentou resultado compatível com a exigência da norma DER-PR ES-P 11/05 (DER-PR, 2005). Esta norma considera que misturas com cimento que apresentem 1,2 $\mathrm{MPa} \leq \sigma_{\mathrm{r}} \leq 2,1 \mathrm{MPa}$ podem ser utilizadas como sub-base e 1,5 MPa $\leq \sigma_{\mathrm{r}} \leq 2,1 \mathrm{MPa}$ podem ser utilizadas como base, ambas definidas na norma como misturas tratadas com cimento. Por outro lado, essa mesma norma considera que misturas com cimento que apresentem $\sigma_{\mathrm{r}}>2,1 \mathrm{MPa}$ são consideradas como do tipo solo-cimento e podem ser utilizadas em camadas de sub-base e base.

Tabela 7 ISC, expansão e RCNC dos materiais e misturas na energia modificada.

\begin{tabular}{lccccc}
\hline Materiais & \% de cimento & $\begin{array}{c}\text { ISC } \\
(\%)\end{array}$ & $\begin{array}{c}\text { Expansão } \\
(\%)\end{array}$ & $\begin{array}{c}\boldsymbol{\sigma}_{\mathbf{r}} \\
(\mathbf{M P a})\end{array}$ & $\begin{array}{c}\text { CV }\left(\boldsymbol{\sigma}_{\mathbf{r}}\right) \\
(\mathbf{\%})\end{array}$ \\
\hline Solo & 0 & 20,20 & 0,05 & 0,37 & - \\
RAP & 0 & 15,00 & 0 & 0 & - \\
$50 \%$ de RAP & 2 & 97,10 & 16,61 \\
$50 \%$ de RAP & 4 & 117,10 & 0 & 0,27 & 22,22 \\
$70 \%$ de RAP & 2 & 83,50 & 0 & 0,43 & 17,14 \\
$70 \%$ de RAP & 4 & 84,50 & 0 & 0,35 & 3,08 \\
$50 \%$ de RAP & 5 & - & 0 & 0,52 & 28,85 \\
\end{tabular}


Vale destacar os elevados valores de CV observados na Tabela 6, com destaque para as misturas com 50\% de RAP e $2 \%$ e $5 \%$ de cimento, onde esse parâmetro estatístico ficou acima de $20 \%$.

\subsection{Avaliação do Solo-RAP como Mistura Solo-cimento}

Estão apresentados na Tabela 8 os resultados dos testes com a mistura contendo 50\% de RAP, para os teores de cimento de $6 \%, 7 \%, 8 \%, 9 \%$ e $10 \%$.
Embora os valores de RCNC tenham crescido com o aumento do teor de cimento, a exigência mínima da norma DNIT ES 143 (DNIT, 2010a) para resistência média à compressão não confinada, de $2,1 \mathrm{MPa}$, não foi atendida por nenhum dos teores testados. Admitindo um crescimento linear da resistência, com o aumento do teor de cimento, seria necessário cerca de $12 \%$ de cimento para atingir esse valor (Figura 4), sendo este teor considerado bastante elevado tanto do ponto de vista econômico, devido ao custo do cimento, quanto técnico, em função do risco de ocorrência de trincas por retração da camada de base.

Tabela 8 ISC, expansão e RCNC do solo e mistura solo-cimento com 50\% de RAP na Energia Normal.

\begin{tabular}{cccc}
\hline Materiais & \% de cimento & $\begin{array}{c}\boldsymbol{\sigma}_{\mathbf{r}} \\
(\mathbf{M P a})\end{array}$ & $\begin{array}{c}\mathbf{C V} \\
\text { (\%) }\end{array}$ \\
\hline Solo & 0 & 0,37 & 0,06 \\
$50 \%$ de RAP & 6 & 0,71 & 25,51 \\
$50 \%$ de RAP & 7 & 0,95 & 2,27 \\
$50 \%$ de RAP & 8 & 1,22 & 4,37 \\
$50 \%$ de RAP & 9 & 1,33 & 2,61 \\
$50 \%$ de RAP & 10 & 1,71 & 8,19 \\
\hline
\end{tabular}

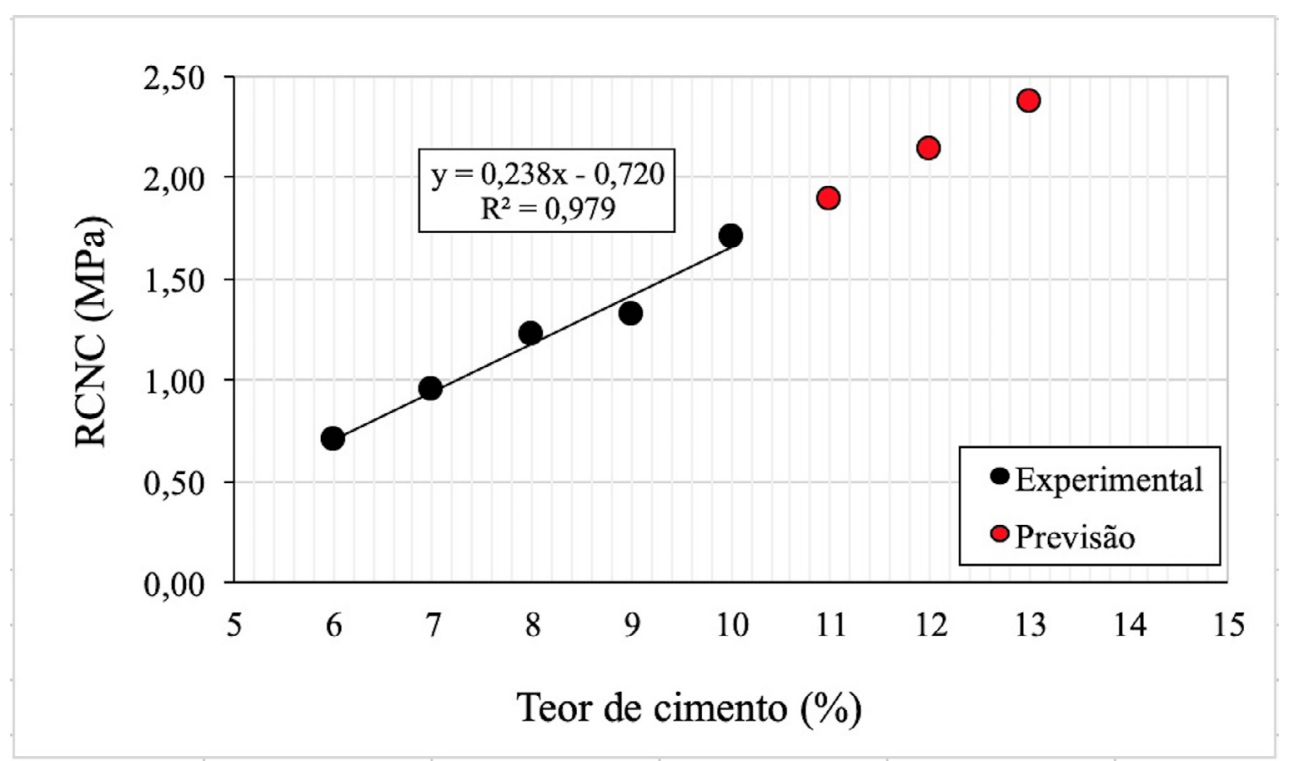

Figura 4 Previsão de alcance da RCNC mínima, de acordo com a DNIT ES 143 (DNIT, 2010a). 
Entretanto, como visto anteriormente, considerando os resultados da Tabela 8, pela norma DER-PR ES-P 11/05 (DER-PR, 2005) apenas a mistura contendo 50\% de RAP e $10 \%$ de cimento se habilitou para uso como base de pavimento. Ainda de acordo com a norma DER-PR ES-P 11/05 (DER-PR, 2005), as misturas com 50\% de RAP e $8 \%$ e $9 \%$ de cimento, entretanto, se habilitaram para uso em camadas de sub-base.

Contudo, estes teores também são considerados elevados, pelos mesmos argumentos anteriormente descritos, prova disso é que a DEINFRA-SC ES-P09/16 (DEINFRA$\mathrm{SC}, 2016)$ limita o teor máximo de cimento em 3\%.

\section{Conclusões}

Os resultados obtidos demonstraram a inviabilidade de uso das misturas solo-RAP sem cimento como camada de base de pavimentos rodoviários, de acordo com a norma DNIT ES 141 (DNIT, 2010c), estando essas misturas aptas, apenas, em alguns teores de solo e RAP (50\% e 70\%) para uso como camada de sub-base, conforme a DNIT ES 139 (DNIT, 2010d).

Quanto à utilização com cimento, as misturas se mostraram promissoras em diferentes teores de cimento para $50 \%$ de adição de RAP, como camada de base, entretanto, a tomada de decisão sobre qual teor adotar dependerá do normativo ou especificação de serviço utilizado no projeto da mistura.

\section{Referências}

ABNT. 2012. Associação Brasileira de Normas Técnicas. NBR 12253 - Solo-cimento - Dosagem para emprego como camada de pavimento. Rio de Janeiro: ABNT, 3p.

ABNT. 2016a. Associação Brasileira de Normas Técnicas. NBR 6459: Solo-Determinação do limite de liquidez. Rio de janeiro: ABNT, 5p.

ABNT. 2016b. Associação Brasileira de Normas Técnicas. NBR 7180: Solo - Determinação do limite de plasticidade. Rio de janeiro: ABNT, 2016b. 3p.

ABNT. 2018. Associação Brasileira de Normas Técnicas. NBR 7181:2016 Versão Corrigida 2:2018. - Solo - Análise granulométrica. Rio de Janeiro: ABNT, 12p.

Adhikari, S.; Khattak, M.J. \& Adhikari, B. 2018. Mechanical characteristics of Soil-RAP-Geopolymer mixtures for road base and subbase layers. International Journal of Pavement Engineering, 21(4): 483-496.

Alhaji, M.M. \& Alhassan, M. 2018. Effect of Reclaimed Asphalt Pavement Stabilization on the Microstructure and Strength of Black Cotton Soil. International Journal of Technology, 4: 727-736.

DEINFRA-SC. 2016. Departamento de Infraestrutura do Estado de Santa Catarina. Pavimentação: Reciclagem profunda de pavimentos. ES-P09/16. Santa Catarina, 21 p.
DER-BA. 2001. Departamento de Estradas de Rodagem do Estado da Bahia. Base de Solo melhorado com cimento. ES-P-06/01. Bahia, 9 p.

DER-PR. 2005. Departamento de Estradas de Rodagem do Estado do Paraná. Pavimentação: solo-cimento e solo tratado com cimento. ES-P 11/05. Paraná, 19 p.

DNER. 1994a. Departamento Nacional de Estradas de Rodagem. ME 93: Solo - Determinação da Densidade Real. Rio de Janeiro, 4 p.

DNER. 1994b. Departamento Nacional de Estradas de Rodagem. ME 258: Solos Compactados com Equipamentos Miniatura - Mini-MCV. Rio de Janeiro, 14 p.

DNER. 1994c. Departamento Nacional de Estradas de Rodagem. ME 256: Solos Compactados com Equipamentos em Miniatura - Determinação da Perda de Massa por Imersão. Rio de Janeiro, 6 p.

DNER. 1994d. 1994d. Departamento Nacional de Estradas de Rodagem. ME 53: Misturas betuminosas - Porcentagem de betume. Rio de Janeiro, 5 p.

DNER. 1994e. Departamento Nacional de Estradas de Rodagem. ME 202: Solo-cimento - Moldagem e cura de corpos de prova cilíndricos, Rio de Janeiro, 7 p.

DNER. 1994f. Departamento Nacional de Estradas de Rodagem. ME 201: Solo-cimento - Compressão axial de corpos de prova cilíndricos. Rio de Janeiro, 4 p.

DNER. 1996. Departamento Nacional de Estradas de Rodagem. CLA 259: Classificação de Solos Tropicais para Finalidades Rodoviárias Utilizando Corpos-de-prova Compactados em Equipamentos Miniatura. Rio de Janeiro, 6 p.

DNER. 1998. Departamento Nacional de Estradas de Rodagem. ME 81: Agregados - Determinação da absorção e da densidade de agregado graúdo. Rio de Janeiro, 6 p.

DNIT. 2010a. Departamento Nacional de Infraestrutura de Transportes. ES 143: Pavimentação - Base de solo-cimento. Rio de Janeiro, $10 \mathrm{p}$.

DNIT. 2010b. Departamento Nacional de Infraestrutura de Transportes. ES 142: Pavimentação - Base de solo melhorado com cimento. Rio de Janeiro, 9 p.

DNIT. 2010c. Departamento Nacional de Infraestrutura de Transportes. ES 141: Pavimentação - Base estabilizada granulometricamente. Rio de Janeiro, 9 p.

DNIT. 2010d. Departamento Nacional de Infraestrutura de Transportes. ES 139: Pavimentação - Sub-base estabilizada granulometricamente. Rio de Janeiro, $8 \mathrm{p}$.

DNIT. 2013. Departamento Nacional de Infraestrutura de Transportes. ME 164: Solo - Compactação Utilizando Amostras não Trabalhadas. Rio de Janeiro, 7 p.

DNIT. 2016. Departamento Nacional de Infraestrutura de Transportes. ME 172: Solo - Determinação do Índice de Suporte Califórnia Utilizando Amostras não Trabalhadas. Brasília, 17 p.

EMBRAPA. 2018. Empresa Brasileira de Pesquisa Agropecuária. Sistema Brasileiro de Classificação de Solos, Brasília, DF, $355 \mathrm{p}$.

Fedrigo, W.; Núñez, W.P.; Ceratti, J.A.P.; Ely, V.; Souza, E.; Gonçalves, G.; Magalhães, A.; Machado Filho, P.; Melo, D.; Gonçalves, B. 2015. Efeito da energia de compactação e 
do teor de fresado no comportamento mecânico de misturas típicas da reciclagem de pavimentos com adição de cimento. Revista Estradas, 14: 22-27.

Gaspar, M.S., Vasconcelos, K.L., Lopes, M.M., Bernucci, L.L.B. 2020. Evaluation of binder blending on warm mix asphalt recycling. Revista Transportes, 28(2): 87-99.

Ghanizadeh, A.L. \& Rahrovan, M. 2019. Modeling of unconfined compressive strength of soil-RAP blend stabilized with Portland cement using multivariate adaptive regression spline. Frontiers of Structural and Civil Engineering, 13: 787-799.

Ghanizadeh, A.L.; Rahrovan, M. \& Bafghi, K.B. 2018. The effect of cement and reclaimed asphalt pavement on the mechanical properties of stabilized base via full-depth reclamation. Construction and Building Materials, 161: 165-174.

Nogami, J.S. \& Villibor, D.F. 1995. Pavimentação de baixo custo com solos lateríticos. São Paulo, Villibor, 213p.

Ochepo, J. 2014. Stabilization of laterite soil using reclaimed asphalt pavement and sugarcane bagasse ash for pavement construction. Journal of Engineering Research, 2(4): 1-13.

Rodrigues, M.G.F.; Nacif, P.G.S; Costa, O.V. \& Olszevski, N. 2009. Solos e suas Relações com as Paisagens Naturais no Município de Cruz das Almas - BA. Revista de Biologia e Ciência da Terra, 9(2): 193-205.
Segundo, I.G.R.; Branco, V.T.F.C.; Vasconcelos, K.L. \& Holanda, A.S. 2016. Misturas asfálticas recicladas a quente com incorporação de elevado percentual de fresado como alternativa para camada de módulo elevado. Revista Transportes, 24(4): 85-94.

Suddeepong, A.; Intra, A.; Horpibulsuk, S.; Suksiripattanapong, C.; Arulrajah, A. \& Shen, J.S. 2018. Durability against wetting-drying cycles for cement-stabilized reclaimed asphalt pavement blended with crushed rock. Soils and Foundations, 58(2): 333-343.

Suebsuk, J.; Suksan, A. \& Horpibulsuk, S. 2014. Strength assessment of cement treated soil-reclaimed asphalt pavement (RAP) mixture. International Journal of Geomate, 6(2): 878-884.

Suebsuk, J.; Horpibulsuk, S.; Suksan, A.; Suksiripattanapong, C.; Phoo-ngernkham, T. \& Arulrajah, A. 2019. Strength prediction of cement-stabilised reclaimed asphalt pavement and lateritic soil blends. International journal of pavement engineering, 20(3): 332-338.

Unger Filho, W.; Klinsky, L.M.G.; Motta, R. \& Bernucci, L.L.B. 2020. Cold Recycled Asphalt Mixture using 100\% RAP with Emulsified Asphalt-Recycling Agent as a New Pavement Base Course. Advances in Materials Science and Engineering, 2020: $1-11$.

\section{How to cite:}

Almeida, V.G.; Almeida, M.S.S.; Maciel, F.C.S.; Moura, L.N.; Costa, W.G.S.; \& São Mateus, M.S.C. 2021. Diferentes Metodologias de Dosagem de Misturas Solo-RAP para Uso em Pavimentação. Anuário do Instituto de Geociências, 44: 36210. DOI 1982-3908_2021_44_36210 